\title{
TETRACYCLINE ABSORPTION AFTER INJURY
}

\author{
Major M.S. OWEN-SMITH, F.R.C.S., F.R.C.S. (Edin.), R.A.M.C. \\ Military Hospital, Terendak
}

SUMMARY: The use of Tetracycline by oral administration as protection against wound infection in missile injuries is discussed. A method of measuring serum concentrations of this antibiotic is described. A comparison is made of the serum levels in normal control patients, with those from patients who had sustained a close fracture of the femur.

Results show that absorption of Tetracycline from the gut is very poor following a moderately severe injury, and that therapeutic serum levels are not obtained.

When military activity occurs in difficult terrain, the problems of casualty collection and evacuation to medical centres are great. Such a situation existed during the period of confrontation between Indonesia and Malaysia in the dense jungles of Sarawak and Sabah. In this type of country the soldier might well suffer a delay of twenty-four hours or more between wounding and operation. This delay allows infection of the wound to become well established with consequent wound sepsis, delayed healing and an increased risk to life or the loss of a limb.

In an attempt to mitigate this increasing risk of sepsis with the passage of time, antibiotics were incroduced into the soldier's personal first aid pack. These were to be taken after wounding and regularly thereafter (Stephen, 1966). The antibiotic was tetracycline and this was chosen for ease of administration, broad spectrum of activity against pathogenic bacteria and lack of immediate side effects.

However, at that time, no evidence existed that the absorption of tetracycline from the gastro-intestinal tract was normal after wounding. Similarly there was no evidence that it was an effective prophylatic against gas gangrene occurring in the high velocity missile wound. Owen-Smith (1967) showed that tetracycline given intramuscularly was completely effective in preventing fatal gas gangrene in sheep that had been shot through the thigh with a high velocity bullet, and the wound deliberately contaminated with spores of Clostridium oedematiens. All untreated animals died of gas gangrene.

The remaining question was whether tetracycline would be absorbed from the gut in sufficient quantity to attain therapeutic blood concentrations after wounding. This paper sets out to investigate the problem.

\section{Method}

Injured patients. In the absence of missile wounds, a major traumatic condition was chosen for study. Five patients who had sustained closed fractures of the shaft of the femur were treated by the insertion of a Steinmann pin through the tibial tubercle and the application of skeletal traction. They all had lacerations or abrasions for which antibiotics were indicated and all started on tetracycline within three hours of injury. Replacement of estimated blood loss, with transfusion of three packs of blood in each case, was commenced at the most within six hours of injury.

Control patients. Five hospital patients who were treated with tetracycline for infections formed the control group.

Tetracycline therapy. Tetracycline (Tetracyn-Pfizer) capsules were given by mouth. A 
loading dose of $500 \mathrm{mg}$ was followed thereafter by $250 \mathrm{mg}$ every six hours. In the case of the injured patients the loading dose was given as soon as practicable after injury.

Serum samples. $10 \mathrm{ml}$ of venous blood was taken into a sterile container at set intervals after tetracycline therapy commenced. A minimum of five samples were taken from each patient; these were at the 3,6, 9, 12 and 24 hour stages. The blood was allowed to clot; when the clot was retracted the samples were centrifuged. The serum was aspirated and stored at minus $20^{\circ} \mathrm{C}$.

Tetracycline assay. A standard, serial double dilution technique for measuring concentration of antibiotic by its activity against a sensitive test organism was used in preliminary studies. This method was not accurate enough and was discarded.

The alternative method, which was adopted, determines the weight of antibiotic present in unit volume of serum. It is based on the method used by Pfizer Ltd and evolved from the work of Lees and Toothill (1955). The technique compares the patient's serum, containing an unknown concentration of tetracycline, with standards containing a known concentration.

Pfizer Ltd kindly provided crystalline tetracycline containing $980 \mu \mathrm{g}$ per mg. From this was made three standards containing $1.0 \mu \mathrm{g}, 0.33 \mu \mathrm{g}$ and $0.11 \mu \mathrm{g}$ tetracycline per $\mathrm{ml}$ of 33 per cent pooled human serum in phosphate buffer $\mathrm{pH} 4.5$. The patients' sera were similarly diluted three fold with phosphate buffer $\mathrm{pH} 4.5$ and again three-fold with a mixture of 33 per cent pooled human serum in phosphate buffer $\mathrm{pH} 4.5$.

Seeded test plates were obtained by adding $1.0 \mathrm{ml}$ of a dilute suspension of spores of Bacillus cereus (NCIB 8172) to a $100 \mathrm{ml}$ bottle of Oxoid agar, and mixing well before pouring the medium into Petri dishes to cool. When the surface was firm, stainless steel cylinders measuring $10 \mathrm{~mm}$ in length and $6 \mathrm{~mm}$ inside diameter were placed upright on the medium, three to a Petri dish. The three standard solutions and the dilutions of the patient's serum were transferred into the cylinders by pipette. The dishes were then incubated for eighteen hours at $37^{\circ} \mathrm{C}$. The diluted serum diffused into the agar from the cylinder and the tetracycline content inhibited the growth of the spores of Bacillus cereus seeded throughout the medium. Thus a circular zone of inhibition of growth was obtained concentric to the circular cylinder. The zone diameter was proportional to the concentration of tetracycline in the serum. The zone diameters for the three standards were plotted against their known concentrations of tetracycline on three cycle log paper. This resulted in a straight line graph. The diameter of the zones of inhibition of the patients' serum were then plotted, and their corresponding concentrations read off the graph directly. The final concentration of tetracycline present in the test sample was obtained by multiplying by the dilution factors, 3 and 9 , and obtaining the mean value. There was close correlation between the results from the two dilutions.

The Minimum Inhibitory Concentration (M.I.C.) of tetracycline measured in $\mu \mathrm{g} / \mathrm{ml}$ was 0.06 for Bacillus cereus (NCIB 8172).

\section{Results}

The serum concentrations of tetracycline obtained in the control group were averaged and are shown in Fig. 1. There was a rapid rise in concentration after the loading dose reaching just under $1.0 \mu \mathrm{g} / \mathrm{ml}$ in one hour. Peak levels were recorded after three hours followed by a gradual fall until the next dose was given after six hours. Thereafter 


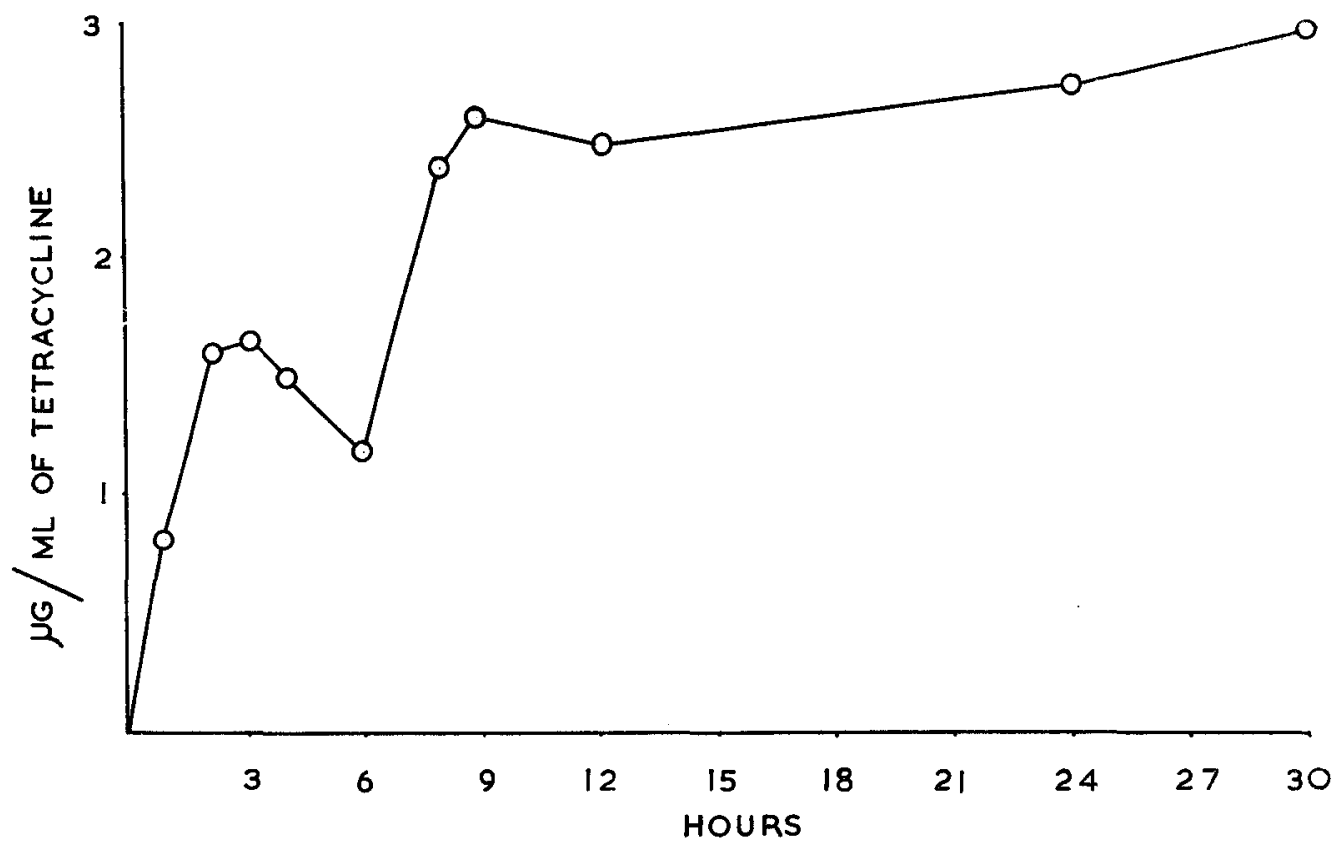

Fig. 1. Average serum concentrations of control patients.

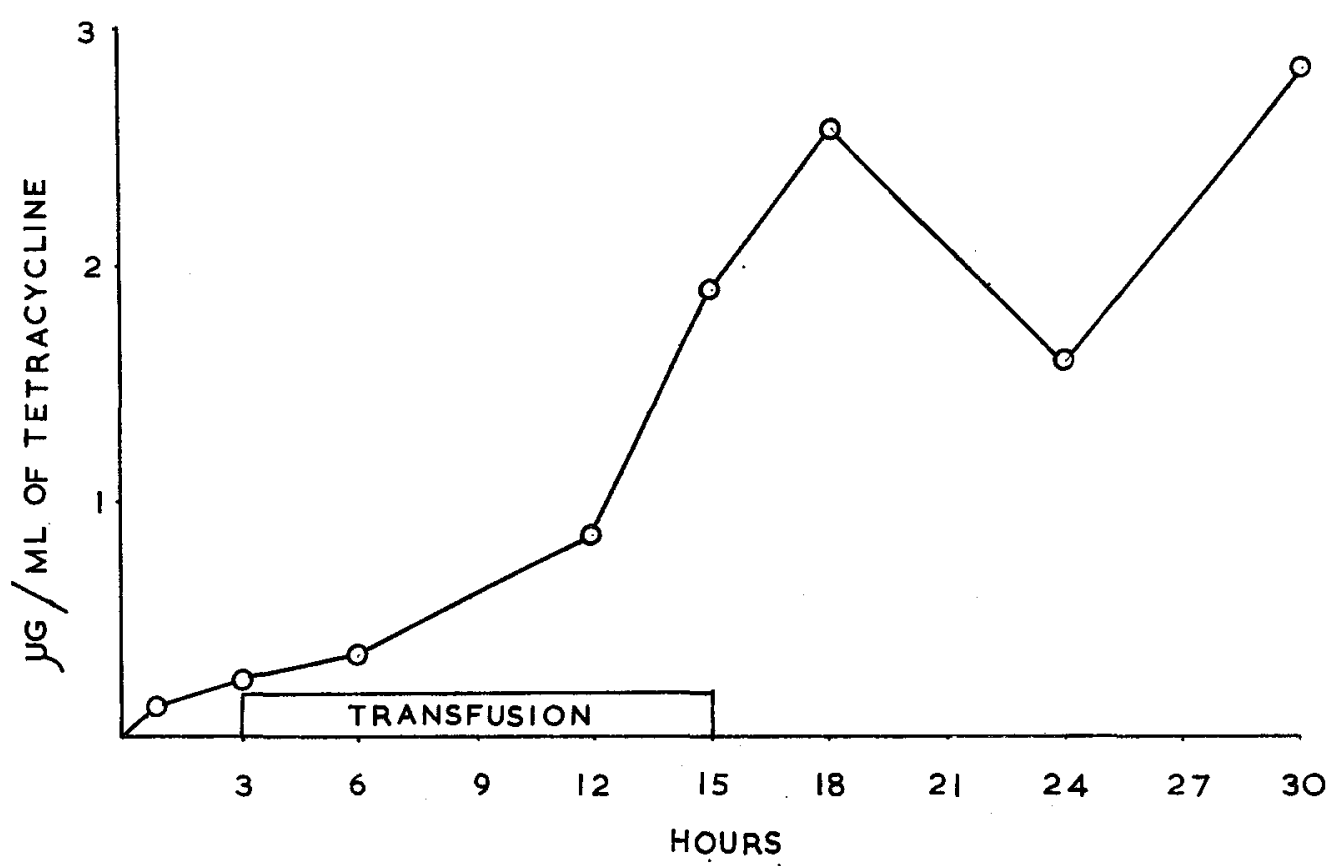

Fig. 2. Average serum concentrations of injured patients. 
the concentrations increased progressively and reached levels of between 3.0 and 4.0 $\mu \mathrm{g} / \mathrm{ml}$ after forty-eight hours. These levels were maintained for the remainder of the drug course.

There was a marked reduction of the serum concentrations of tetracycline obtained from the injured patients (Fig. 2). Concentrations remained low for the first six hours and it was not until twelve hours that therapeutic concentrations were obtained. By this time the shock had been treated by blood transfusion. Thereafter there was a rapid rise so that by eighteen hours the curve approached that of the controls and was almost normal later.

In one other patient with a fractured femur the transfusion of four packs of blood was completed within twelve hours of injury. Tetracycline was then commenced orally and blood samples taken thereafter at the usual intervals. The concentrations obtained are shown in Fig. 3. There was a more pronounced rise in serum concentration during

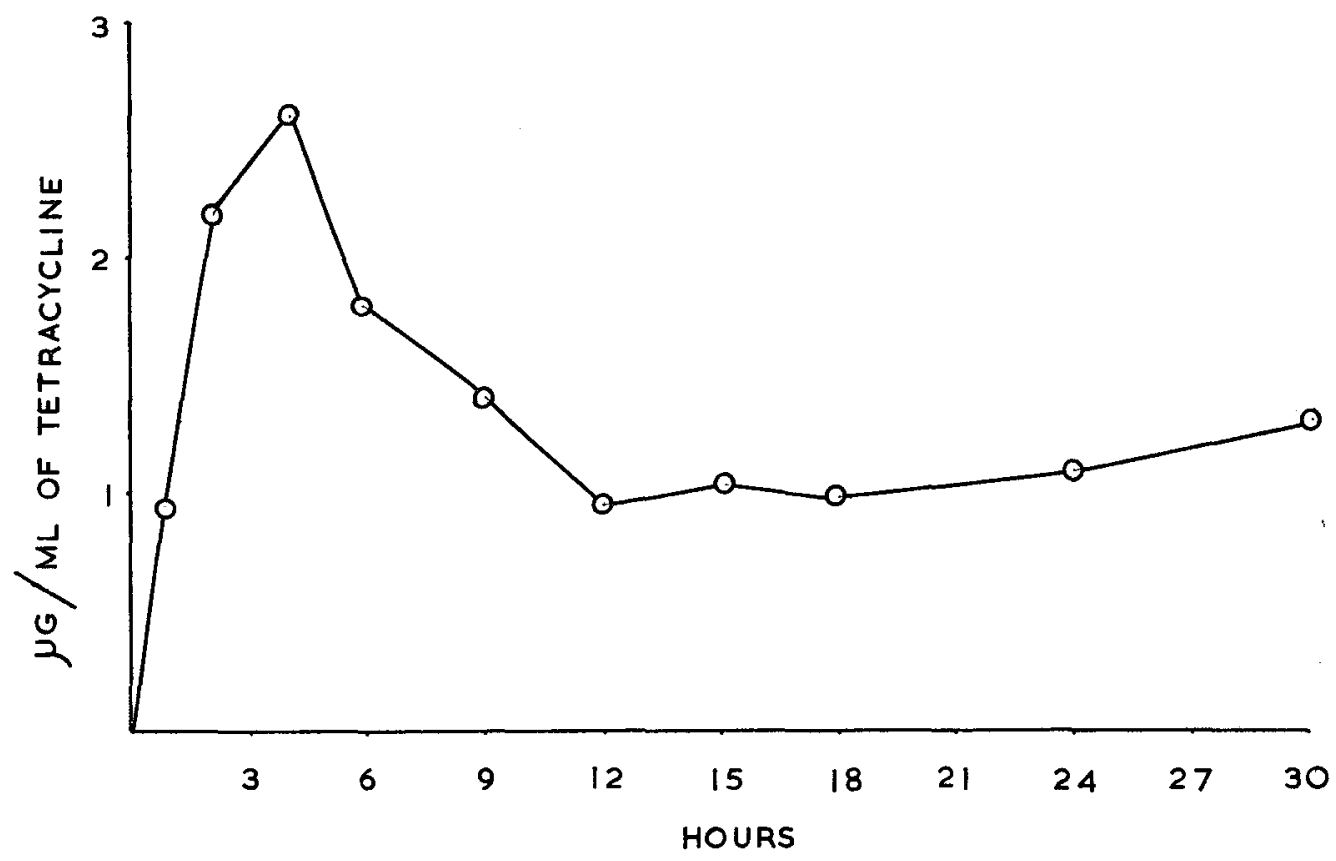

Fig. 3. Serum concentrations obtained when tetracycline was commenced after the completion of blood transfusion in an injured patient.

the first six hours compared with the normal controls. The curve then gradually returned to normal over the next twenty-four hours.

\section{Discussion}

Wheatley (1967) gave details from the Borneo campaign, in which there was no orthodox line of evacuation through Regimental Aid Post and Advanced Dressing Station. Casualties were picked up by helicopter and brought to Kuching hospital where 
the Field Surgical Team was situated. Delays of twenty-four hours or more, due to bad weather or darkness, often occurred. 70 per cent of all patients took longer than six hours before being given surgical treatment. Although sepsis occurred in nearly 40 per cent of all cases, and was relatively more common in the severely injured patients, overall he considered that sepsis was not an important cause of morbidity or mortality. An analysis of the incidence of sepsis in relation to the delay between wounding and operation suggested to him that in moderate wounds thirty-six hours may elapse with comparative safety under antibiotic cover, but that in severe wounds, and those involving bone, it was imperative to operate within six hours of injury.

However, the Borneo figures show that the effect of self-administration of tetracycline in preventing sepsis appeared to be negligible (Table 1). It may be that the

Table 1

Sepsis rates of severe and moderate wounds in the Borneo campaign (Wheatley, 1967)

\begin{tabular}{l|c|c|c}
\multicolumn{1}{c|}{ Prophylactic antibiotic } & Number & Sepsis & Rate per cent \\
\hline Tetracycline & 28 & 10 & 35 \\
Penicillin and Streptomycin & 12 & 6 & 50 \\
Nil & 56 & 19 & 34 \\
\hline
\end{tabular}

patients with severe wounds developed sepsis because the tetracycline taken orally was not absorbed to any extend due to shock, and therefore adequate therapeutic blood levels were not obtained. We know that the major portion of absorbed tetracycline leaves the gastro-intestinal tract from the stomach and duodenum. (Herrell, Heilman and Wellman, 1950, Pindell, Cull, Doran and Dickinson, 1959). Absorption from the stomach and duodenum is by a simple diffusion process which depends on the concentration gradient established between the lumen of the gut and mucosa. The greater the local blood flow, the more rapidly will tetracycline be absorbed and carried away by the blood to the rest of the tissues. There the gradient is reversed. Consequently blood returning to the stomach will have a low concentration and will take up more from the gastric mucosa. In shock, one of the compensatory mechanisms to keep an adequate blood flow to the essential organs is the constriction of the blood vessels of the splanchnic circulation as a result of sympathetic activity. Consequently, the gastric blood fiow in shock is greatly reduced, the concentration gradient for absorption of tetracycline is low, and blood levels attained remain low until gastric blood flow is increased. Further practical proof of the direct relationship between blood flow and serum concentrations is shown in Fig. 3. These samples were obtained from a patient with a fractured femur who was rapidly transfused with four packs of blood. Clinically he had a hyperdynamic circulation twelve hours after injury, when the transfusion was completed and tetracycline therapy commenced. The increased absorption from the stomach and duodenum in the first six hours, compared with the normal controls, is shown, and is almost certainly a reflection of the increased gastric blood flow.

The M.I.C. of tetracycline measured in $\mu \mathrm{g} / \mathrm{ml}$ for Clostridia is 0.03 to 0.25 , whereas for Staphylococci it is 0.1 to 0.2 and for Escherichia coli it is as high as $1.0 \mu \mathrm{g} / \mathrm{ml}$ (Barber 
and Garrod, 1963). Thus it can be seen from Fig. 1 that in the normal subject good therapeutic blood levels are obtained after oral administration of tetracycline. Fig. 2 reveals that after an injury, comparable in severity to a high velocity missile wound of the thigh, oral tetracycline results in blood levels which barely attain the M.I.C. for Clostridia, Staphylococci and Streptococci. It is well recognised that therapeutic blood levels should be at least two to three times the M.I.C. of the infecting organism. Only after the commencement of blood transfusion does the serum concentration rise to an effective level in the wounded patients. In the normal patient serum concentration of tetracycline definitely increases as the individual dose is raised from $250 \mathrm{mg}$ to $1.0 \mathrm{~g}$, but above that dose level increased oral doses produce little corresponding increase in the serum level of antibiotic (Sayer, Michel, Moll and Kirby, 1951); therefore the ways to increase the serum concentration after oral tetracycline in the presence of shock would be to increase the gastric blood flow, or increase the individual dose. The first would necessitate blood transfusion to establish an adequate circulating blood volume, and would not be practicable in the circumstances envisaged. The second would be very limited by the poor gastric blood flow, but nevertheless by this method it might just be possible to double the present low serum concentration and thus attain minimal therapeutic levels.

Further experiments are being done on this particular aspect, in an attempt to produce a suitable regime which would be effective under the conditions of shock.

\section{Conclusion}

This study suggests that oral tetracycline is not effective in shocked subjects. It indicates that those soldiers with severe and moderate wounds would obtain little therapeutic benefit from the oral self administration of tetracycline tablets. An alternative method of treatment must be found to protect such patients.

\section{Acknowledgements}

My thanks are due to Dr. G. M. Williamson and Mr. K. Horn of the Therapeutics Research Division of Pfizer L.td, for their great help in the planning and execution of this project.

\section{REFERENCES}

Barber, M. and Garrod, L. P. (1953). Antibiotics and Chemotheraphy. E. \& S. Livingstone, Edinburgh. LeEs, K. A. and Toothill, J. P. R. (1955) Analyst 80, 95, 110 and 531.

Herrell, W. E., Hetrman, F. R. and Wellanan, W. E. (1950). Ann. N. Y. Acad. Sci. 53, 448.

OWEN-SMTT, M. S. (1968). Brit. J. Surg. 55, 43.

Pindeil, M. H., CUll, K. M., Doran, K. M. and Drckinson, H. L. (1959). J. Pharmacol. exp. Ther. $125,287$.

SAYer, R. J., Michel, J. C., Moll, F. C. and Kirby, W. M. M. (1951). Amer. J. med. Sci. 221, 256.

STEPHEN, R. A. (1966). Director of Army Surgery. Directive No. 3.

WhEATLEY, P. R. (1967). J. roy. Army med. Cps 113, 18. 